\title{
Mesoscopic fluctuations of the Coulomb drag
}

\author{
B. N. Narozhny and I. L. Aleiner \\ Department of Physics and Astronomy,SUNY Stony Brook, Stony Brook, NY 11794 \\ Theoretische Physik III, Ruhr-Universität Bochum, 44780 Bochum, Germany
}

\begin{abstract}
We consider mesoscopic fluctuations of the Coulomb drag coefficient $\rho_{D}$ in the system of two separated two-dimensional electron gases. It is shown that at low temperatures sample to sample fluctuations of $\rho_{D}$ exceed its ensemble average. It means that in such a regime the sign of $\rho_{D}$ is random and the temperature dependence almost saturates $\rho_{D} \sim 1 / \sqrt{T}$.
\end{abstract}

PACS numbers: 73.23.-b, 73.50.-h, 73.61.-r

When two electronic layers are brought close to each other to form a bi-layer system, a current flowing through one of the layers(the active layer) is known to induce a voltage $V_{D}=\rho_{D} I$ in the other (passive) layer [1 1 . The effect, which is called the drag, was first predicted theoretically [1,2] in the model where the carriers in the two spatially separated layers interacted via longrange Coulomb interaction. Experimentally the Coulomb drag was first observed in a three-dimensional electron gas layer while the current was driven through a twodimensional electron gas (2DEG) 33. Subsequent experiments studied the effect in 2DEG bi-layers 断, electronhole [5], and normal-metal-superconductor systems [6]. More recently, the drag was studied in the 2DEG bi-layer system in high magnetic field [7].

The quantity, which is studied theoretically is the transconductance $\sigma_{D}$. To the lowest non-vanishing order in the interlayer interaction it is proportional to the drag coefficient $\rho_{D}\left(\sigma_{i}\right.$ is the Drude conductance of the $i$-th layer)

$$
\rho_{D} \approx \frac{\sigma_{D}}{\sigma_{1} \sigma_{2}}
$$

As a function of temperature the observed $\sigma_{D}$ roughly follows the quadratic law $\sigma_{D} \sim T^{2}$, although the ratio $\sigma_{D} / T^{2}$ deviates from the constant value [1, ].

The $T^{2}$ dependence of the Coulomb drag coefficient follows from the Fermi liquid phase space argument. To create a current in the passive layer, it is necessary to create a pair of electron-like (filled states with energy greater than the Fermi energy $\epsilon>\epsilon_{F}$ ) and hole-like excitations (empty states $\epsilon<\epsilon_{F}$ ) in a state with nonzero momentum. The energy and momentum of the pair come from an electron in the active layer, which is moving with the driving current. In each layer, the scattering states are limited to the energies of order $T$ relative to the Fermi level, which gives two powers of $T$ to the drag coefficient. However, the momentum is transferred equally to electrons and holes, therefore in the case of electron-hole symmetry the drag of the electrons cancels that of the holes. Thus the effect is non-zero only due to the electron-hole asymmetry. Similarly, the asymmetry is necessary for the electron and hole system in the active layer to have non-zero total momentum in the first place. The asymmetry can be expressed as a derivative of the density of states $\nu$ and/or the diffusion constant $D$ with respect to the chemical potential $\mu$. This can be obtained rigorously in the diagrammatic formalism [10]. For the case of diffusive layers the disorder-averaged transconductance is

$$
\left\langle\sigma_{D}\right\rangle=\frac{e^{2}}{\hbar} \frac{\pi^{2}}{3} \frac{(\hbar T)^{2}}{g^{2}(\kappa d)^{2}}\left(\frac{\partial}{\partial \mu}(\nu D)\right)^{2} \ln \frac{T_{0}}{2 T},
$$

where for simplicity we take the layers to be identical, so that they have the same chemical potential, diffusion constant and the dimensionless conductance $g=$ $25.8 k \Omega / R_{\square}$. The logarithm is cut at the scale $T_{0}=D \kappa / d$ and $\kappa=2 \pi e^{2} \nu$ is the inverse Thomas-Fermi screening length.

Such effects of the electron-hole asymmetry are wellknown, for instance the thermopower in disordered electronic systems [11] or adiabatic pumping [12]. As these effects are due to the electron-hole asymmetry, the average quantities are small, since each derivative with respect to the chemical potential brings one power of the Fermi energy $E_{F}$ to the denominator. On the other hand, the typical energy scale of mesoscopic effects is the Thouless energy $E_{T}=\hbar D / L^{2}$ ( $L$ is the sample size), which is much smaller than the Fermi energy. Therefore the effects mentioned above exhibit mesoscopic fluctuations, much larger than the average.

In this Letter we show that the mesoscopic fluctuations of the Coulomb drag coefficient can indeed be larger than the average Eq. (11), even if the electron systems in both layers are good metals $(g \gg 1)$. To characterize the fluctuations, we calculate the average square of the (random) transconductance. The result of lengthy albeit straightforward calculations shown below is given by

$$
\begin{aligned}
& \left\langle\sigma_{D}^{\alpha \beta} \sigma_{D}^{\alpha^{\prime} \beta^{\prime}}\right\rangle=\left(\delta^{\alpha \alpha^{\prime}} \delta^{\beta \beta^{\prime}}+\delta^{\alpha \beta^{\prime}} \delta^{\alpha^{\prime} \beta}\right)\left\langle\sigma_{D}^{2}\right\rangle, \\
& \left\langle\sigma_{D}^{2}\right\rangle=\frac{\gamma}{18 \pi^{3}}\left(\frac{32 \ln 2-14}{3}\right) \frac{e^{4}}{\hbar^{2}} \frac{E_{T} \tau_{\varphi} \ln \kappa d}{g^{4}(\kappa d)^{3}},
\end{aligned}
$$

where the numerical factor $\gamma=1.0086$ is the value of the integral 


$$
\begin{gathered}
\gamma=\frac{1}{2} \int_{0}^{\infty} \frac{d x_{1} d x_{2} x_{1} x_{2}}{\left(x_{1}^{2}+x_{2}^{2}\right)}\left[J_{0}\left(x_{1}\right) J_{0}\left(x_{2}\right)+J_{2}\left(x_{1}\right) J_{2}\left(x_{2}\right)\right] \\
\left(K_{0}\left(x_{1}\right) K_{0}\left(x_{2}\right)+\left[\frac{2}{x_{1}^{2}}-K_{2}\left(x_{1}\right)\right]\left[\frac{2}{x_{2}^{2}}-K_{2}\left(x_{2}\right)\right]\right),
\end{gathered}
$$

where $J_{i}(x)$ and $K_{i}(x)$ are the Bessel functions [13]. Here $\tau_{\varphi} \ll\left(E_{T}\right)^{-1}$ is the dephasing time. This result is valid in the most relevant regime $L_{\varphi}=\sqrt{D \tau_{\varphi}} \leq L$ and $\kappa d \gtrsim 1$. If $\kappa d \leq 1$, then the average square of the conductance is $\left\langle\sigma_{D}^{2}\right\rangle \propto \frac{e^{4}}{\hbar^{2}} \frac{E_{T} \tau_{\varphi}}{g^{4}}$ with the coefficient of order unity. In what follows we first discuss the experimental consequences of our results, then explain it qualitatively and finally give the rigorous calculation. The fluctuations Eq. (2) depend on temperature only through the dephasing time $1 / \tau_{\varphi} \approx T / g$ and at low enough temperatures they should dominate the behavior of the transconductance. Therefore the $T^{2}$ decrease of $\sigma_{D}$ should at some small temperature $T_{*}$ be almost saturated at a sampledependent value. Let us estimate $T_{*}$ for the samples used in existing experiments [4, [] using the reported parameters of the samples. Collecting the numerical factors, we write the ratio of the square of the average transconductance Eq. (11) and the averaged square Eq. (2) as

$$
\frac{\left\langle\sigma_{D}\right\rangle^{2}}{\left\langle\sigma_{D}^{2}\right\rangle}=\left(\frac{g T}{E_{F}}\right)^{4} \frac{1}{E_{T} \tau_{\varphi}} \frac{20}{\kappa d \ln \kappa d} .
$$

We take the interlayer spacing to be $d=200 \AA$ [画]; the screening length in GaAs is $\kappa^{-1}=100 \AA$; the Thouless energy is given by $E_{T}=g /\left(2 \pi \nu L^{2}\right)$; and the dephasing time $\tau_{\varphi}^{-1} \simeq T \ln g / g$ [15]. Then we estimate $T_{*}$ as the temperature at which the ratio $\left\langle\sigma_{D}\right\rangle^{2} /\left\langle\sigma_{D}^{2}\right\rangle$ is equal to unity

$$
T_{*}=E_{F}\left(16 \pi g^{2} n L^{2}\right)^{-1 / 5} \approx 0.2 K,
$$

where the Fermi energy in the samples [4, 7] $E_{F} \simeq 60 \mathrm{~K}$, the electron density $n=1.5 \times 10^{11} \mathrm{~cm}^{-2}$, the size of the sample $L \simeq 200 \mu \mathrm{m}$, and the conductance is calculated from the sheet resistance of the sample $R=10 \Omega / \square$.

The estimated $T_{*}$ is lower than the temperature range for the existing data [4], therefore there is no trace of the fluctuations Eq. (2) in the data. However, if one takes a dirtier sample, with the sheet resistance, for instance, $1 k \Omega / \square$, then the estimate for $T_{*}$ becomes $2 K$ and the effect of the fluctuations becomes observable. To push $T_{*}$ even higher, the sample size can be also reduced.

Let us now explain Eq. (2) qualitatively. First, consider the lowest temperature regime $T \ll E_{T}$, so that the sample is effectively zero-dimensional (0D). The mesoscopic fluctuations of the usual conductance are universal $\delta \sigma \simeq e^{2} / \hbar$. The transconductance is associated with the interlayer interactions, thus possessing additional smallness. The value of such smallness can be estimated by the Golden rule argument which is comprised by (i) phase volume; (ii) matrix elements; (iii) electron-hole asymmetry (dependence of the density of states on the energy). Matrix elements in 0D do not depend on energy and give smallness $1 / g^{2}$ 14. Therefore the phase volume is limited by temperature only, which gives the factor $T^{2}$. Finally, the electron-hole asymmetry $\partial_{\mu}(\ln \nu(\mu))$ is the random quantity with the typical value $1 / E_{T}$. Putting everything together, we arrive to the estimate

$$
\text { r.m.s. } \delta \sigma_{D} \sim \frac{e^{2}}{\hbar} \frac{T^{2}}{\left(E_{T} g\right)^{2}}
$$

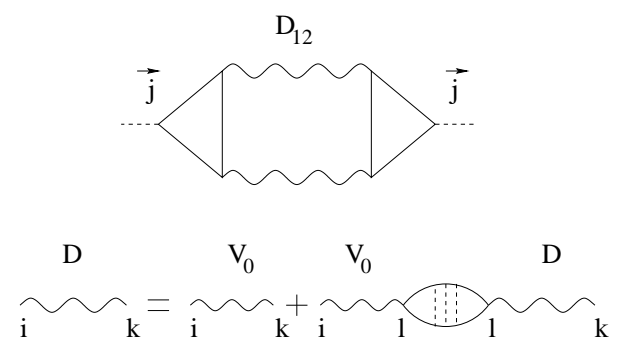

FIG. 1. A. The diagram for the transconductance in the lowest non-vanishing order in the interlayer interaction. The solid lines are the exact Green's functions of the non-interacting electron system (in the presence of disorder) and the wavy lines are the disorder averaged interlayer Coulomb interaction propagators Eq. (6). B. The RPA scheme for the calculation of the interaction propagators. The dashed lines denote the disorder. The indices $i, k, l=1,2$ indicate the layer.

At higher temperatures the averaging should be performed by dividing the sample into patches of the size $L_{\varphi} \times L_{\varphi}$, since on larger scales the phase coherence is destroyed. The contribution of each patch $\left\langle\delta \sigma_{D}^{2}\left(L_{\varphi}\right)\right\rangle$ is approximately the same and they can be simply comboned as a network of random resistors

$$
\left\langle\delta \sigma_{D}^{2}\right\rangle=\left\langle\delta \sigma_{D}^{2}\left(L_{\varphi}\right)\right\rangle\left(\frac{L_{\varphi}}{L}\right)^{2}
$$

Now, $\left\langle\delta \sigma_{D}^{r m s}\left(L_{\varphi}\right)\right\rangle$ can be found similar to Eq. (3) with two important differences: (i) the fluctuations of the density of states are summed from the scale of order $T$, rather than $E_{T}\left(L_{\varphi}\right)$. This suppresses the fluctuations in each layer by the factor $\sqrt{E_{T}\left(L_{\varphi}\right) / T}$. (ii) The matrix elements become energy dependent on the energy scale larger than $E_{T}$, decreasing with the transmitted energy $\omega$ as $|M|^{2} \sim 1 / \omega^{2}$. As a result, the transmitted energy is limited by $\omega \sim E_{T}\left(L_{\varphi}\right)=1 / \tau_{\varphi}$, rather then by $T$, so in the estimate of the phase volume we should replace $T^{2}$ by $T / \tau_{\varphi}$. So, we find

$$
\delta \sigma_{D}\left(L_{\varphi}\right) \sim \frac{T\left(\frac{1}{\tau_{\varphi}}\right)}{\left(E_{T}\left(L_{\varphi}\right) g\right)^{2}}\left(\sqrt{\frac{E_{T}\left(L_{\varphi}\right)}{T}}\right)^{2} \sim g^{-2}
$$


Finally, to estimate the total magnitude of the transconductance fluctuations we substitute Eq. (5) into Eq. (4) to obtain

$$
\left\langle\delta \sigma_{D}^{2}\right\rangle \sim g^{-4}\left(\frac{L_{\varphi}}{L}\right)^{2} \sim g^{-4} E_{T} \tau_{\varphi}
$$

This estimate yields the same result as Eq. (2) up to numerical factors and the dependence on $\kappa d$.

Our results suggest the following picture of the Coulomb drag. If one starts measuring the drag coefficient at high $T$ and proceeds by lowering the temperature, then at first the transresistance will decrease roughly as $T^{2}$, as follows from Eq. (11). At the temperature $T_{*}$, estimated above, the transresistance will appear to saturate $\left(\sigma_{D} \propto 1 / \sqrt{T}\right)$, as the fluctuations Eq. (2) will start to dominate. The particular value of the prefactor will be sample dependent and, what is more important, will have random sign. If the temperature will be decreased further, then at very low temperatures $T<E_{T}$ the sample will effectively become zero dimensional and the $T^{2}$ decrease will be restored (also with a random coefficient), so that at $T=0$ the drag coefficient vanishes.

Let us now present the calculation. The electrons in both layers interact via the Coulomb interaction. The interaction propagators corresponding to the dynamically screened Coulomb interaction can be obtained within the RPA scheme (see Fig. 1B) using the Green's functions of non-interacting electrons. For our purposes we only need the propagator of the interlayer interactions, which is given by (here we set the layers to be identical, so that they have the same density of states $\nu$ and diffusion coefficient $D$ )

$$
\mathcal{D}^{R}(\omega, Q)=\frac{1}{2 \nu D Q^{2}} \frac{\left(-i \omega+D Q^{2}\right)^{2}}{-i \omega+(1+\kappa d) D Q^{2}} .
$$

The transresistance in the disordered two-layer system can be expressedin terms of the exact Green's functions of non-interacting, disordered electron system and the interaction propagators Eq. (6). To the lowest non-vanishing order in the interlayer interaction the transresistance is given by the diagram Fig. 1A. The left and right triangles correspond to the two layers in the system and the wavy line is the interlayer interaction propagator Eq. (6). As the electron Greens's functions now depend on disorder, this $\sigma_{D}$ is a random quantity and its moments should be averaged over disorder. Before averaging, the expression for $\sigma_{D}$ corresponding to the diagram on Fig. 11 can be written as

$$
\sigma_{D}^{\alpha \beta}=\frac{1}{4 V} \int \frac{d \omega}{2 \pi}\left(\frac{\partial}{\partial \omega} \operatorname{coth} \frac{\omega}{2 T}\right) \mathcal{D}_{12}^{R} \Gamma_{23}^{\alpha} \mathcal{D}_{34}^{A} \Gamma_{41}^{\beta},
$$

where the indices indicate the spatial coordinates. Points 1, 2 belong to one layer and 3,4 to the other. The triangular vertices $\Gamma^{\alpha}$ are given by

$$
\Gamma_{12}^{\alpha}(\omega)=\int \frac{d \epsilon}{2 \pi}\left[J_{12}^{\alpha}(\omega, \epsilon)+J_{21}^{\alpha}(-\omega, \epsilon)+I_{12}^{\alpha}(\omega, \epsilon)\right],
$$

where

$$
\begin{array}{r}
J_{12}^{\alpha}(\omega, \epsilon)=\left(\tanh \frac{\epsilon-\omega}{2 T}-\tanh \frac{\epsilon}{2 T}\right) \\
{\left[G_{12}^{R}(\epsilon-\omega)-G_{12}^{A}(\epsilon-\omega)\right]\left[G^{R}(\epsilon) j^{\alpha} G^{A}(\epsilon)\right]_{21} ;} \\
I_{12}^{\alpha}(\omega, \epsilon)=\left(\tanh \frac{\epsilon-\omega}{2 T}-\tanh \frac{\epsilon}{2 T}\right)\left(r_{1}-r_{2}\right)^{\alpha} \\
{\left[G_{12}^{R}(\epsilon) G_{21}^{R}(\epsilon-\omega)-G_{12}^{A}(\epsilon) G_{21}^{A}(\epsilon-\omega)\right] .}
\end{array}
$$

The exact electronic Green's functions used in Eq. (8) can be written in terms of the exact wavefunctions of the system as

$$
G_{12}^{R(A)}(\epsilon)=\sum_{j} \frac{\Psi_{j}^{*}\left(\vec{r}_{1}\right) \Psi_{j}\left(\vec{r}_{2}\right)}{\epsilon-\epsilon_{j} \pm \imath 0}
$$

where $j$ labels the exact eigenstates of the system and $\epsilon_{j}$ are the exact eigenvalues.

The known result for the averaged transconductance Eq. (11) is obtained [10] by averaging the triangular vertices $\Gamma^{\alpha}$ independently for each layer (for the effect of the correlated disorder see Ref. [16]). However, as we discussed above, in the intermediate temperature range the fluctuations exceed the average, and the temperature dependence saturates. To characterize the fluctuations we average the square of the transconductance. The mesoscopic fluctuations of the interaction propagators can be neglected, because they produce only the small fluctuating coefficient in Eq. (7). Therefore, we have to average the product of two triangular vertices $\Gamma^{\alpha}$ (in the same layer; note that in this temperature regime Eq. (8d) does not contribute). The corresponding diagrams are shown in Fig. 2. After averaging, each diagram contains six diffusons and one Hikami box (see Fig. 3). The calculation of the arising 14-dimensional integral is greatly simplified by the following observation. The dominant contribution to the frequency integrals Eq. (8a) comes from the region, where the external frequencies [which are the frequencies of the interaction propagators Eq. (6)] $] \omega_{1}+\omega_{2} \simeq \kappa d / \tau_{\varphi}$ and $\omega_{1}-\omega_{2} \simeq 1 / \tau_{\varphi}$ so that the frequency difference is small compared to the sum. The momentum integral is dominated by the region $1 / \tau_{\varphi} \leq Q \leq \kappa d / \tau_{\varphi}$. Since we are in the intermediate temperature regime $T>E_{T}$, the energy transfer $\omega$ is smaller than the temperature, and the vertices Eq. (8) can be expanded in the inverse temperature. Now the dimensional analysis gives the resulting expression for the fluctuations Eq. (2), which depends on temperature only through $\tau_{\varphi}$ as we discussed above. 
The numerical coefficient can now be obtained by performing the integration without further approximations. The factor $\gamma$ comes from the angle integration and the numerical factor in Eq. (2b) from the integration over the small frequency difference.

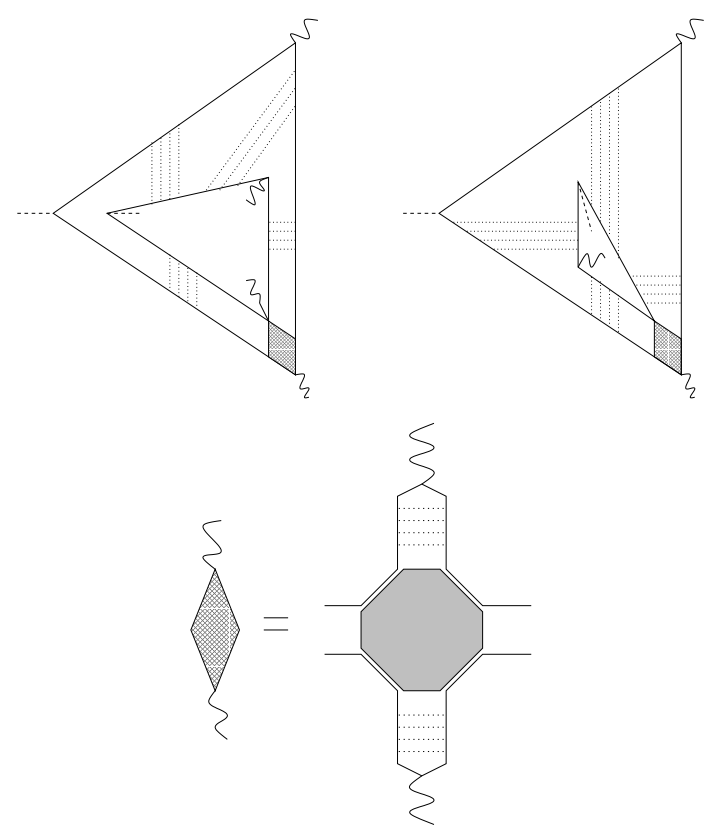

FIG. 2. Diffuson contribution to the average of the product of two triangular vertices. Cooperon contribution is obtained by interchanging of vertices $\rho$ and $j$ in one of the triangles (the direction of arrows should be changed respectively)

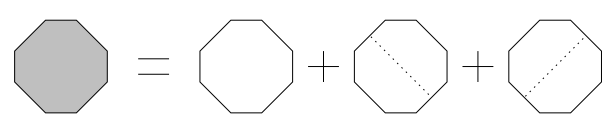

FIG. 3. Hikami box.

In conclusion, we have described the mesoscopic fluctuations of the Coulomb drag coefficient or the transconductance. The fluctuations are characterized by the average square of the random, disorder dependent transconductance Eq. (2). Compared to the averaged transconductance Eq. (11) the fluctuations Eq. (2) are determined by the Thouless energy, rather than the Fermi energy, as the average. Therefore, there exists an intermediate temperature regime, where the fluctuations are greater than the average, which results in the weak $(1 / \sqrt{T})$ temperature dependence of the transconductance in this regime. Moreover, in this regime the $\sigma_{D}$ is a random, sample dependent quantity, so that the sign of the measured value is also random. Since the average transconductance Eq. (11) grows as $T^{2}$, at higher temperatures $\left(T>T_{*}\right)$ the fluctuations are small and the the measured $\sigma_{D}$ is roughly equal to the average. This was the case in the existing experiments [3] mated the crossover temperature $T_{*} \approx 0.2 K$, which was below the temperature range used in these experiments. To observe the effect of the fluctuations, one needs to take a dirtier sample of smaller size. Then $T_{*}$ can be equal to several Kelvin, and the saturation of $\sigma_{D}$ to a value with the random sign can be observed.

Finally, we notice that the Coulomb drag coefficient may also be presented as the product of two random numbers $\rho_{D} \simeq a_{1} a_{2}$, where $a_{1}, a_{2}$ characterize each layer. If the disorder is corelated, the average $\left\langle a_{1} a_{2}\right\rangle$ appears, which leads to the results of Ref. 16. In this respect, the results of Ref. [16] are just a particular manifestation of the mesoscopic fluctuations of $\rho_{D}$, discussed in this Letter.

We acknowledge helpful conversations with B.L. Althsuler, Fei Zhou, and especially with A. Kamenev. The work at Ruhr-Universität Bochum was supported by SFB 237 "Unordnung and grosse Fluctuationen". I.A. is A.P. Sloan and Packard research fellow.

[1] M.B. Pogrebinskii, Fiz. Tekh. Poluprovodn. 11, 637 (1977).

[2] P.M. Price, Physica (Amsterdam) 117B, 750 (1983).

[3] P.M. Solomon, P.J. Price, D.J. Frank, and D.C. La Tulipe, Phys. Rev. Lett. 63, 2508 (1989).

[4] T.J. Gramila, J.P. Eisenstein, A.H. MacDonald, L.N. Pfeiffer, and K.W. West, Phys. Rev. Lett. 66, 1216 (1991).

[5] U. Sivan, P.M. Solomon, and H. Shtrikman, Phys. Rev. Lett. 68, 1196 (1992).

[6] N. Giordano and J.D. Monnier, Phys. Rev. B, 50, 9363 (1994).

[7] M.P. Lilly,J.P. Eisenstein, L.N. Pfeiffer, and K.W. West, Phys. Rev. Lett. 80, 1714 (1998).

[8] T.J. Gramila, J.P. Eisenstein, A.H. MacDonald, L.N. Pfeiffer, and K.W. West, Phys. Rev. B, 47, 12957 (1993).

[9] H.C. Tso, P. Vasilopoulos, and F.M. Peeters, Phys. Rev. Lett. 68, 2516 (1992).

[10] A. Kamenev and Y. Oreg, Phys. Rev. B, 52, 7516,(1995)

[11] A.V. Anisovich, B.L. Altshuler, A.G. Aronov, and A.Yu. Zyuzin, Pis'ma Zh. Eksp. Teor. Fiz. 45, 237 (1987) [Sov. Phys. JETP. Lett. 45, 295 (1987)].

[12] Fei Zhou, B.L. Altshuler, and B.Z. Spivak, Phys. Rev. Lett. 82, 608 (1999).

[13] M. Abramowitz and I. Stegun, Handbook of Mathematical Functions (Dover, New York, 1972).

[14] I.L Aleiner, B.L. Altshuler, and M.E. Gershenson, Waves in Random Media, (1999).

[15] B.L. Altshuler and A.G. Aronov, in Electron-Electron Interactions in Disordered Systems,eds. A.L. Efros, M. Pollak (North-Holland, Amserdam, 1985).

[16] I.V. Gornyi, A.G. Yashenkin, and D.V. Khveshchenko, Phys. Rev. Lett. 83, 152 (1999). 